\title{
A follow up study of myocardial involvement in patients with mitochondrial encephalomyopathy, lactic acidosis, and stroke-like episodes (MELAS)
}

\author{
Y Okajima, Y Tanabe, M Takayanagi, H Aotsuka
}

Division of Cardiology, Chiba Children's Hospital, Chiba City, Japan

Y Okajima

H Aotsuka

Division of Neurology, Chiba Children's Hospital

Y Tanabe

Division of

Metabolism, Chiba Children's Hospital M Takayanagi

Correspondence to: Dr Yoshitomo Okajima, Division of Cardiology, Chiba Children's Hospital, 579-1 Heta-chou, Midori-ku, Chiba City 266-0007, Japan.

Accepted for publication 30 April 1998

\begin{abstract}
Objective-To investigate cardiac function in patients with mitochondrial encephalomyopathy, lactic acidosis, and stroke-like episodes (MELAS) and clarify the clinical features of cardiomyopathy in MELAS. Patients-11 consecutive patients with MELAS (mean age at initial examination 11.3 years, range 4 to 16 years) were enrolled in the study. Six were followed for more than five years.

Results-On echocardiographic examination, three patients showed increased left ventricular end diastolic posterior wall thickness (LVPWTd), exceeding $140 \%$ of the normal value. Four patients, including these three, had an ejection fraction of less than $50 \%$, and two also had increased left ventricular end diastolic volume (LVEDV) exceeding $140 \%$ of the normal value $(\% \mathrm{~N})$. The LVPWTd\%N was correlated positively with the LVEDV\%N $(R=0.669$, $p<0.05)$ and negatively with the ejection fraction $(R=-0.6701, p<0.05)$. One patient died of heart failure aged 22 years. Conclusions-The cardiomyopathy in MELAS is characterised by an abnormally thick left ventricular wall with progressive dilatation and poor left ventricular contraction developing over several years, indicating hypertrophic cardiomyopathy advancing to dilated cardiomyopathy.

(Heart 1998;80:292-295)

Keywords: MELAS; cardiomyopathy
\end{abstract}

Table 1 Results of genetic and cardiac analyses in patients with MELAS

\begin{tabular}{|c|c|c|c|c|c|}
\hline Case & Sex & $\begin{array}{l}\text { Age at final } \\
\text { examination } \\
\text { (years) }\end{array}$ & $\begin{array}{l}\text { mtDNA } \\
\text { mutation }\end{array}$ & ECG findings & UCG diagnosis \\
\hline 1 & $\mathrm{~F}$ & 23 & 3243 & $\begin{array}{l}\text { Intermittent WPW; } \\
\text { inverted T wave in II, III, } \\
\text { aVF, V5, V6; }\end{array}$ & $\mathrm{HCM}$ \\
\hline 2 & $\mathrm{~F}$ & 22 (lost) & 3243 & $\begin{array}{l}\text { Inverted } \mathrm{T} \text { wave in III, } \\
\text { V6 }\end{array}$ & $\mathrm{HCM} \rightarrow \mathrm{DCM}$ \\
\hline 3 & M & 21 & 3243 & WPW & $\mathrm{HCM} \rightarrow \mathrm{DCM}$ \\
\hline 4 & $\mathrm{M}$ & 6 & 3243 & WPW & Normal \\
\hline 5 & $M$ & 8 & 3243 & Flat $\mathrm{T}$ wave in V5-V6 & Normal \\
\hline 6 & M & 7 & 3243 & $\begin{array}{l}\text { ST-T down slope at first, } \\
\text { then normalised }\end{array}$ & $\begin{array}{l}\text { Initially LV dilatation; } \\
\text { during follow up, normal } \\
\text { size }\end{array}$ \\
\hline 7 & M & 15 & 3471 & Normal & Not done \\
\hline 8 & $M$ & 22 & 3243 & Inverted $\mathrm{T}$ wave in aVL & Normal \\
\hline 9 & $\mathrm{~F}$ & 11 & 3243 & Normal & Not done \\
\hline 10 & M & 17 (lost) & 3243 & $\begin{array}{l}\text { Inverted } \mathrm{T} \text { wave in II, } \\
\text { III, aVF, V5, V6 }\end{array}$ & Normal \\
\hline 11 & $M$ & 13 & 3243 & PVC & Normal \\
\hline
\end{tabular}

DCM, dilated cardiomyopathy; HCM, hypertrophic cardiomyopathy; LV, left ventricle; mtDNA, mitochondrial DNA; MELAS, mitochondrial encephalomyopathy, lactic acidosis, and stroke-like episodes; PVC, premature ventricular contractions; UCG, ultrasound cardiogram; WPW, WolffParkinson-White syndrome.
Cardiomyopathy is known to be one of the important complications of several types of mitochondrial disease. ${ }^{1-11}$ In patients with mitochondrial encephalomyopathy, lactic acidosis, and stroke-like episodes (MELAS), cardiomyopathy - especially hypertrophic cardiomyopathy (HCM) - is known to be common. ${ }^{8-11}$ Some patients manifest cardiomyopathy with poor ventricular contraction, and this is thought to be one of the causes of death at the advanced stage of the disease. ${ }^{13}$ However, neither the clinical condition nor its course has been fully assessed. In this study, we investigated cardiac function in patients with MELAS during subsequent follow up.

\section{Methods}

Eleven consecutive patients with MELAS were enrolled in the study (table 1). All gave their informed consent before participating in the study. Their mean age was 11.3 years (range 4 to 16 ) at the initial examination, and there were no patients in whom the onset was after adulthood. The mean follow up period was 6.9 years (range 2 to 10 years), and six patients were followed up for more than five years.

The symptoms at onset were headache or seizure in seven patients, delayed motor development in two, mental retardation in one, and easy fatiguability in one. At follow up, recurrent stroke was observed in four patients, and one showed quadriplegia. Two patients were bedridden because of severe muscle weakness or dementia at the advanced stage of the disease.

Mitochondrial DNA analysis was carried out by polymerase chain reaction on muscle biopsy specimens. ${ }^{12-14}$ Ten patients showed point mutation of 3243 and the other of 3471 in the mitochondrial DNA analysis. The diagnosis of MELAS was confirmed in all patients based on their clinical symptoms.

\section{EVALUATION OF CARDIAC FUNCTION}

Cardiac function was analysed using electrocardiography (ECG), chest $x$ ray, and echocardiography. Echocardiography was performed in nine patients but not the other two because their heart sounds, ECG, and chest $x$ ray were all normal. The end diastolic and end systolic left ventricular volumes (LVEDV and LVESV, respectively) were measured by the area-length method, and the ejection fraction and left ventricular end diastolic posterior wall thickness (LVPWTd) were obtained.

The data for echocardiography were compared with the normal values at our institution based on an equation using body surface 
Table 2 Final echocardiography data

\begin{tabular}{|c|c|c|c|c|c|c|}
\hline Case & $B S A\left(m^{2}\right)$ & $\begin{array}{l}\text { LVEDV } \\
(\mathrm{ml})\end{array}$ & $L V E D V \% N$ & $L V E F$ & $\begin{array}{l}\text { LVPWTd } \\
(\mathrm{mm})\end{array}$ & LVPWTd\%N \\
\hline 1 & 1.11 & 51 & 63.4 & 63.4 & 11.4 & 160 \\
\hline 2 & 1.30 & 191 & 194.9 & 24.7 & 18.0 & 237 \\
\hline 3 & 1.17 & 120 & 140.3 & 27.2 & 10.4 & 143 \\
\hline 4 & 0.95 & 58 & 89.5 & 59.8 & 7.2 & 108 \\
\hline 5 & 0.72 & 52 & 116.1 & 49.3 & 5.8 & 98 \\
\hline 6 & 0.69 & 55 & 128.4 & 39.0 & 6.5 & 112 \\
\hline 8 & 1.68 & 167 & 120.6 & 59.8 & 10.4 & 123 \\
\hline 10 & 0.80 & 36 & 70.0 & 66.1 & 5.7 & 92 \\
\hline 11 & 0.96 & 33 & 50.9 & 66.7 & 7.0 & 105 \\
\hline Mean (SD) & $1.04(0.32)$ & $85(59)$ & $108.2(45.1)$ & $50.7(16.5)$ & $9.2(4.0)$ & $131(45)$ \\
\hline
\end{tabular}

BSA, body surface area; LVEDV, left ventricular end diastolic volume; LVEDV $\% \mathrm{~N}$, percentage of normal value for left ventricular end diastolic volume; LVEF, left ventricular ejection fraction; LVPWTd, left ventricular posterior wall thickness; LVPWTd\%N, percentage of normal value for left ventricular posterior wall thickness.

area, ${ }^{15}{ }^{16}$ and expressed as percentages of the normal values $(\% \mathrm{~N}) .^{15}$

To evaluate ventricular size or wall thickness in paediatric patients, it is important to normalise the data because body mass in this age group is quite variable, especially during follow up. We therefore used normalised equations for patients at our institution, ${ }^{15}$ which enabled us to compare cardiac size among patients of different ages or body masses and to assess the degree of abnormal deviation objectively.

\section{Results}

ECG FINDINGS

Six patients showed depolarisation abnormalities, such as inverted $\mathrm{T}$ wave in leads II, III, aVF, and V5-V6. Three showed WolffParkinson-White (WPW) syndrome. One showed premature ventricular contraction. Nine of the 11 patients underwent echocardiography.

ECHOCARDIOGRAPHIC FINDINGS

The data for the most recent echocardiographic examination are summarised in table 2. The mean LVEDV was $85 \mathrm{ml}$ (range 33 to $191 \mathrm{ml}$ ) and the mean (SD) LVEDV\%N was 108.2 (45.1)\% (range $50.9 \%$ to $194.9 \%$ ). Two patients showed increased LVEDV exceeding $140 \%$ of the normal value. The mean (SD) ejection fraction was 50.7 (16.5)\% (range
$24.7 \%$ to $66.7 \%$ ). The mean LVPWTd was 9.2 $\mathrm{mm}$ (range 5.7 to $18.0 \mathrm{~mm}$ ) and the mean (SD) LVPWTd $\% \mathrm{~N}$ was 131 (45)\% (range $92 \%$ to $237 \%$ ); three patients showed increased LVPWTd\%N exceeding $140 \%$. Four patients, including these three, showed decreased ejection fraction values of less than $50 \%$.

The LVPWTd $\% \mathrm{~N}$ was correlated positively with the LVEDV $\% \mathrm{~N}(\mathrm{y}=0.665 \mathrm{x}+21.2, r=$ $0.669, \mathrm{p}<0.05)$ and negatively with the ejection fraction $\left(\mathrm{y}=101 \mathrm{e}^{(-0.0057 \mathrm{x})}, r=-0.6701\right.$, $\mathrm{p}<0.05)$ (fig 1).

\section{RESULTS OF FOLLOW UP}

During the follow up of six patients for more than five years (mean 6.9 years), three (patients 1,2 , and 3 ) showed a worsening ejection fraction of less than $50 \%$ and an increased LVPWTd $\% \mathrm{~N}$ and LVEDV $\% \mathrm{~N}$ exceeding $140 \%$ within several years (fig 2). In patient 6 , who had congestive heart failure at the onset, cardiac function improved with a decrease in LVEDV $\% \mathrm{~N}$ from $141 \%$ to $116 \%$ after initiation of treatment with digitalis, diuretics, and vasodilators. His condition has remained stable during follow up, although the ejection fraction has been gradually worsening with an increase of LVPWTd\%N.

Two patients have died, one from encephalopathy and the other, patient 2 , from congestive heart failure. In this case, the echocardiographic findings had shown severe left ventricular wall hypertrophy $(237 \%$ of the normal value), enlargement $(194.9 \%$ of the normal value), and poor contraction (ejection fraction $24.7 \%$ ) (fig 3 ).

\section{Discussion}

Cardiomyopathy is a common cardiac manifestation in MELAS-like conduction abnormalities such as the Wolff-Parkinson-White syndrome, ${ }^{11}$ and it has been categorised as hypertrophic on the basis of morphological assessment with echocardiography and necropsy examination. ${ }^{1-11}$ In idiopathic hypertrophic cardiomyopathy, left ventricular contractility usually remains within the normal
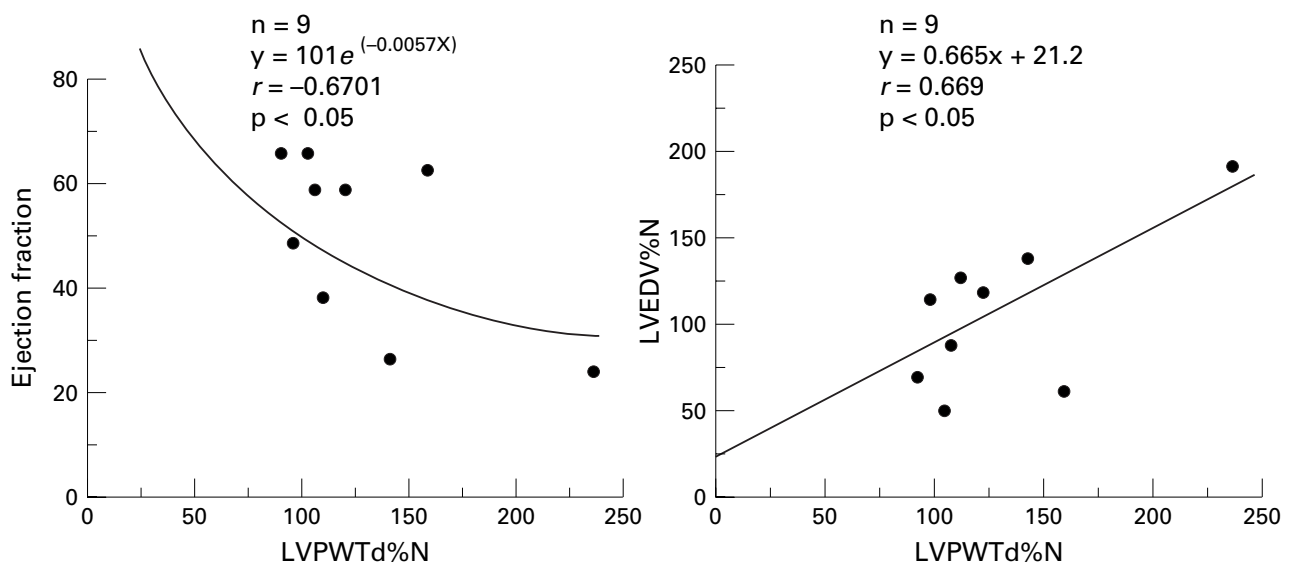

Figure 1 Correlation of percentages of the normal values of left ventricular posterior wall thickness (LVPWTd\%N) with ejection fraction (left), and with percentages of the normal values of left ventricular end diastolic volume (LVEDV\%N) (right). The LVPWTd\%N showed significant negative correlation with ejection fraction and a positive correlation with $L V E D V \% N$ 
range. Sudden cardiac arrhythmia is the major cause of death and only $10-15 \%$ of these patients show transition to dilated cardiomyopathy. ${ }^{17} 18$
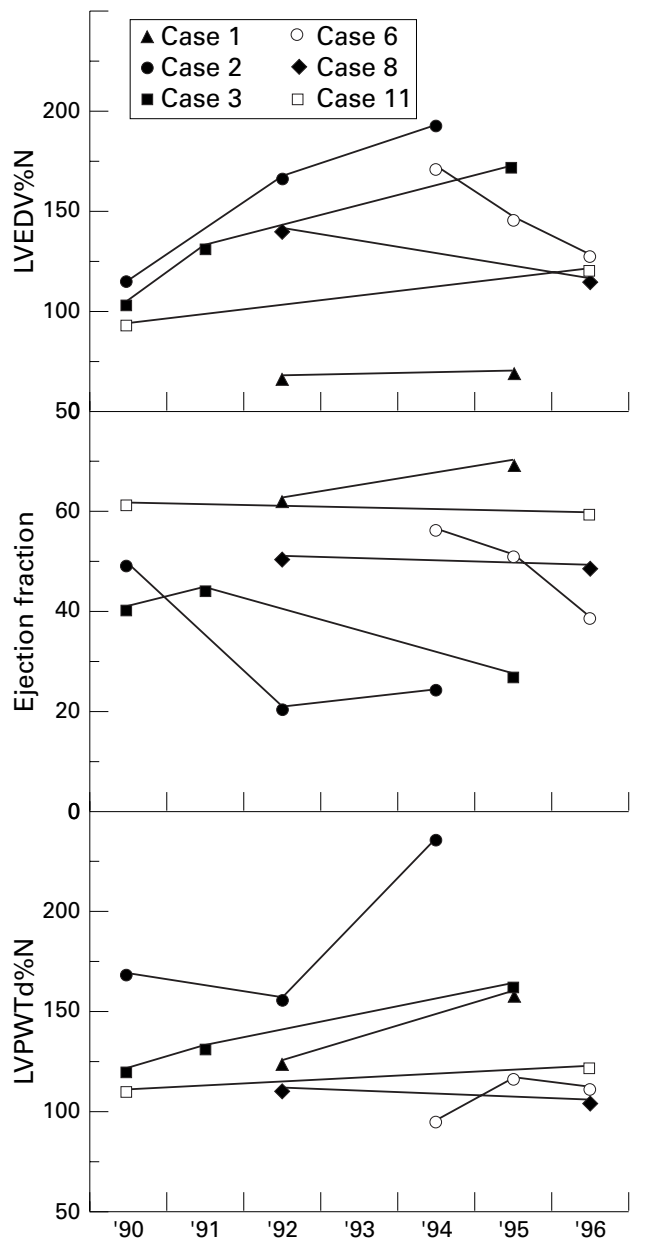

Figure 2 Follow up results for left ventricular end diastolic volume (LVEDV\%N), ejection fraction, and left ventricular posterior wall thickness (LVPWTd\%N) in six patients. Patients 1, 2, and 3 showed left ventricular wall hypertrophy, and 2 and 3 showed deterioration of left ventricular contraction during follow up.

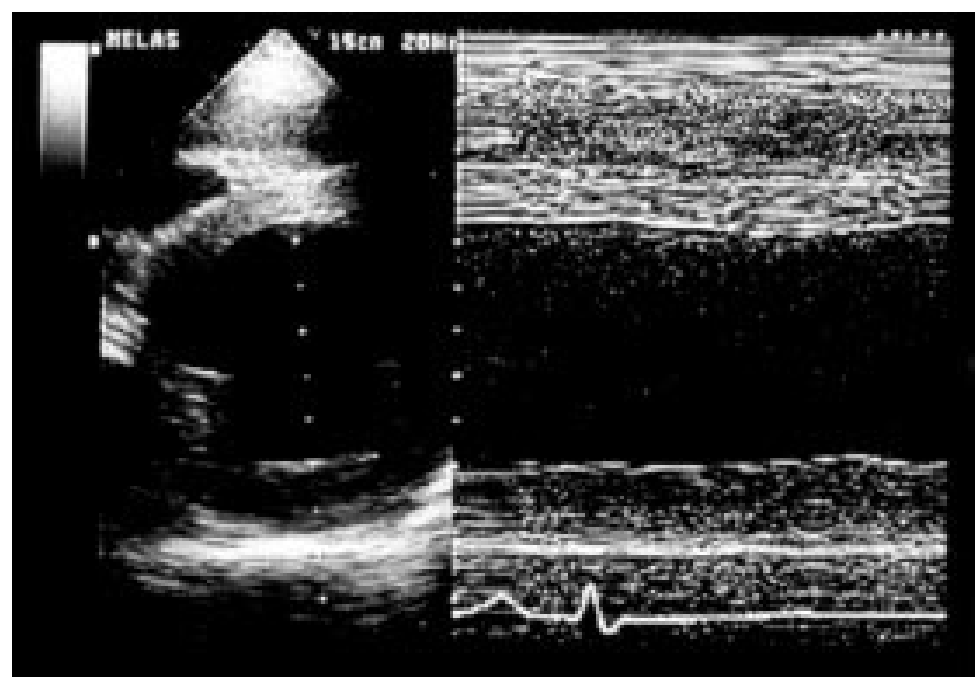

Figure 3 Echocardiography in patient 2, which showed severe hypertrophy of the left ventricular wall and poor contraction. She died of heart failure.
Although, cardiac dysfunction resembling dilated cardiomyopathy has sometimes been observed in MELAS, ${ }^{13}$ a change in the condition from hypertrophic to dilated cardiomyopathy has not been described. In MELAS with cardiac manifestation, as in idiopathic hypertrophic cardiomyopathy, left ventricular wall thickness gradually increases. With advancing hypertrophy, however, cardiac function gradually deteriorates until cardiac dilatation occurs as a result of an increase in left ventricular end diastolic volume and a decrease in the ejection fraction, while wall thickness steadily increases. We found a positive correlation of LVPWTd with LVEDV\% $\mathrm{N}$ and a negative correlation with ejection fraction in these patients, and there was an association between increased wall thickness and impaired systolic function. These findings are characteristic of the cardiomyopathy in MELAS and have not been described in patients with hypertrophic cardiomyopathy secondary to the sarcomeric protein mutations.

The causes of the progression of hypertrophic cardiomyopathy to dilated cardiomyopathy have not been established, although several studies have shown that myocardial ischaemia is involved in hypertrophic cardiomyopathy ${ }^{19-22}$ and the progression was observed after wall thinning had begun.

The mechanism of cardiac deterioration in patients with MELAS is thought to be the degeneration of cardiac muscle fibres ${ }^{6}$ and microangiopathy detected using ${ }^{201} \mathrm{Tl}$ scintigraphy. ${ }^{23}$ It has been reported that the presence of interstitial fibrosis in cardiomyocytes in MELAS is a point of resemblance between the cardiomyopathy of MELAS and idiopathic hypertrophic cardiomyopathy. ${ }^{4}{ }^{6}$ In other reports the abnormalities have been described as diffuse vacuolar degeneration of cardiomyocytes detectable by light microscopy, and a marked increase of aggregates of enlarged mitochondria with proliferated cristae seen on electronmicroscopy ${ }^{9}$; these findings were very different from those in idiopathic hypertrophic cardiomyopathy. The vacuolisation in the cardiomyocyte might result in the muscle fibre hypertrophy and morphological hypertrophic cardiomyopathy, eventually leading to hypertrophic dilated cardiomyopathy.

It is usually difficult to improve cardiac function once the deterioration has begun. One of our patients, however, responded well to conventional treatment for heart failure. Accordingly, it should be emphasised that routine checks of cardiac function are essential for the follow up of patients with MELAS and that treatment should be started without delay when the clinical presentation deteriorates.

Although the clinical diagnosis of MELAS in our patients had already been confirmed before the study, there are reports of patients with mitochondrial disorders in whom the cardiac manifestation is the only abnormality. ${ }^{4-6}$ The characteristic echocardiographic findings such as wall thickening and decreased left ventricular contraction, together with characteristic ECG depolarisation abnormalities, may 
suggest the diagnosis of MELAS; other features include a family history of short stature, diabetes mellitus, abnormal renal function, hearing loss, and other disorders.

The aetiology of the hypertrophic cardiomyopathy of MELAS syndrome has not been fully established, and the diagnosis is based solely on the morphology of the ventricular wall thickness. However, it is clear that the clinical course is quite dependent on the pathogenesis of the disease. We suggest that it is important to confirm whether this involves mitochondrial disorders or other abnormalities. The underlying causes of myocardial hypertrophy remain unanswered in conditions where there are mutations of sarcomeric protein or mitochondrial DNA. This hypertrophy might induce degeneration of cardiac function. An understanding of the pathogenesis of the myocardial hypertrophy may allow the disease process to be modified in hypertrophic cardiomyopathy.

1 Ito T, Hattori K, Tanaka M, et al. Mitochondrial cytopathy.

Jpn Circ f 1990;54:1214-20.
2 Nishizawa M, Tanaka K, Shinozawa K, et al. A mitochonNishizawa $M$, Tanaka $\mathrm{K}$, Shinozawa $\mathrm{K}$, et al. A mitochondrial encephalomyopathy with cardiomyopathy: a case
revealing a defect of complex I in the respiratory chain. $f$ revealing a defect of complex I

3 Yoneda M, Tanaka M, Nishikimi M, et al. Pleiotropic molecular defects in energy-transducing complex in mitochondrial encephalomyopathy (MELAS). $\mathscr{f}$ Neurol Sci 1989;92:143-58

4 Hattori K, Ogawa T, Kondo T, et al. Cardiomyopathy with mitochondrial DNA mutations. Am Heart f 1991;122:8669.

5 Zeviani M, Gellera C, Antozzi C, et al. Maternally inherited myopathy and cardiomyopathy: associated with mutation in mitochondrial DNA tRNA ${ }^{\text {Leu(UUR) }}$. Lancet 1991;338:1437 .

6 Obayashi T, Hattori K, Sugiyama S, et al. Point mutation in mitochondrial DNA in patients with hypertrophic cardiomyopathy. Am Heart fु 1992;124:1263-9.

7 Matthews PM, Hopkin J, Brown JB, et al. Comparison of the relative levels of the 3243 (A-G) mtDNA mutation in heteroplasmic adult and fetal tissues. F Med Genet 1994;31: 41-4.
8 Maneuver S, Rotig A, Hannebique G, et al. Point mutation of the mitochondrial tRNA ${ }^{\text {Leu }}$ gene (A $3243 \mathrm{G}$ ) in maternally inherited hypertrophic cardiomyopathy, maternally inherited hypertrophic cardiomyopathy, diabetes mellitus, renal failu

fMed Genet 1995;32:654-6.
9 Hiruta Y, Chin K, Shitomi K, et al. Mitochondrial encephalopathy with $A$ to $G$ transition of mitochondrial transfer $\mathrm{RNA}^{\mathrm{Leu}(\mathrm{UUR})} 3243$ presenting hypertrophic cardiomyopathy. Intern Med 1995;34:670-3.

10 Kovalenko S, Tanaka M, Yoneda $\mathrm{M}$, et al. Accumulation of somatic nucleotide substitutions in mitochondrial DNA associated with the $3243 \mathrm{~A}-$-to- $\mathrm{G}$ tRNA ${ }^{\mathrm{Leu}(\mathrm{UUR})}$ mutation in encephalomyopathy and cardiomyopathy. Biochem Biophys Res Commun 1996;222:201-7.

11 Anan R, Nakagawa M, Miyata M, et al. Cardiac involvement in mitochondrial diseases - a study of 17 patients with documented mitochondrial DNA defects. Circulation 1995; 91:955-61.

12 Goto Y, Nonaka I, Horai S. A mutation in the tRNA ${ }^{\mathrm{Leu}(\mathrm{UUR})}$ gene associated with the MELAS subgroup of mitochongene associated with the MELAS subgroup of mit
drial encephalomyopathies. Nature 1990;348:651-3.

13 Kobayashi Y, Momoi MY, Tominaga K, et al. A point mutation in the mitochondrial tRNA ${ }^{\text {Leu(UUR) }}$ gene in MELAS (mitochondrial myopathy, encephalopathy, lactic acidosis and stroke-like episodes). Biochem Biophys Res Commun 1990;173:816-22.

14 Goto Y, Nonaka I, Horai S. A new mtDNA mutation associated with mitochondrial myopathy, encephalopathy, lactic acidosis and stroke-like episodes (MELAS). Biochim Biophys Acta 1991;1097:238-40.

15 Aotsuka H, Tateno S, Hamada $\mathrm{H}$, et al. An exponential growth model for nonlinear regression analysis between diverse cardiac measurements and body size. Fpn f Pediatr 1995;99:1756-64. [In Japanese with English abstract.]

16 Gutgesell HP, Rembold CM. Growth of the heart relative to body surface area. Am f Cardiol 1990;65:662-8.

17 Spirito P, Bellone P. Natural history of hypertrophic cardiomyopathy. Br Heart f 1994;72:S10-12.

18 Maron BJ, Cecchi F, McKenna WJ. Risk factor for sudden cardiac death in patients with hypertrophic cardiomyopacardiac death in patients with hyp
thy. Br Heart $\mathcal{F} 1994 ; 72:$ S13-18.

19 Pasternac A, Noble J, Steulens Y, et al. Pathophysiology of chest pain in patients with cardiomyopathies and normal coronary arteries. Circulation 1982;65:778-89.

20 Cannon RO, Rosing DR, Maron BJ, et al. Myocardial ischemia in patients with hypertrophic cardiomyopathy: contribution of inadequate vasodilator reserve and elevated left ventricular filling pressures. Circulation 1985;71:23443.

21 O'Gara PT, Bonow RO, Maron BJ, et al. Myocardial perfusion abnormalities in patients with hypertrophic cardiomyopathy: assessment with thallium-201 emission computed tomography. Circulation 1987;76:1214-23.

22 Maron BJ, Wolfson JK, Epstein SE, et al. Intramural ("small vessel") coronary artery disease in hypertrophic cardiomyopathy. 7 Am Coll Cardiol 1986;8:545-57.

23 Sato W, Tanaka M, Sugivama S, et al. Cardiomyopathy and angiopathy in patients with mitochondrial myopathy, angiopathy in patients with mitochondrial myopathy, Am Heart f 1994;128:733-41. 